\title{
Secure Layered Transmission in Multicast Systems with Wireless Information and Power Transfer
}

\author{
Derrick Wing Kwan Ng*, Robert Schober ${ }^{\dagger}$, and Hussein Alnuweiri ${ }^{\ddagger}$ \\ Institute for Digital Communications, Friedrich-Alexander-University Erlangen-Nürnberg (FAU), Germany*† \\ Texas A\&M University at Qatar, Qatar ${ }^{\ddagger}$ \\ Email: kwan@lnt.de, schober@lnt.de, hussein.alnuweiri@qatar.tamu.edu
}

\begin{abstract}
This paper considers downlink multicast transmit beamforming for secure layered transmission systems with wireless simultaneous information and power transfer. We study the power allocation algorithm design for minimizing the total transmit power in the presence of passive eavesdroppers and energy harvesting receivers. The algorithm design is formulated as a nonconvex optimization problem. Our problem formulation promotes the dual use of energy signals in providing secure communication and facilitating efficient energy transfer. Besides, we take into account a minimum required power for energy harvesting at the idle receivers and heterogeneous quality of service (QoS) requirements for the multicast video receivers. In light of the intractability of the problem, we reformulate the considered problem by replacing a non-convex probabilistic constraint with a convex deterministic constraint which leads to a smaller feasible solution set. Then, a semidefinite programming relaxation (SDR) approach is adopted to obtain an upper bound solution for the reformulated problem. Subsequently, sufficient conditions for the global optimal solution of the reformulated problem are revealed. Furthermore, we propose two suboptimal power allocation schemes based on the upper bound solution. Simulation results demonstrate the excellent performance and significant transmit power savings achieved by the proposed schemes compared to isotropic energy signal generation.
\end{abstract}

\section{INTRODUCTION}

In recent years, using multimedia applications over wireless communication channels such as internet protocol television (IPTV) and video streaming has become increasingly popular. The associated high data rate requirements have led to a tremendous demand for energy and bandwidth. As a result, advanced signal processing techniques for multiple antenna transmitters and physical layer multicasting have been proposed in the literature for facilitating power efficient communication services [1]-[3]. However, the lifetime of networks remains the bottleneck for system performance. In particular, mobile devices are often powered by capacity-limited batteries and energy is dissipated even if the receivers are idle.

The integration of energy harvesting technology with communication devices provides self-sustainability to powerconstrained communication networks. Hydroelectric, piezoelectric, solar, and wind are the major conventional energy sources for energy harvesting. However, the availability of these natural energy sources is usually limited by location or climate and they may not be available in indoor environments. On the other hand, wireless power transfer, where energy is harvested from ambient radio signals in radio frequency (RF), is also a viable source of energy for energy scavenging [4]-[7]. Furthermore, wireless energy harvesting technology facilitates the possibility of simultaneous wireless information and power transfer. In [4] and [5], the fundamental tradeoff between the harvested energy and the achievable data rate was investigated for flat

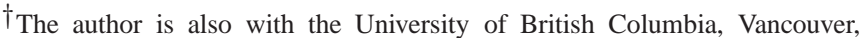
Canada. This research was supported by the Qatar National Research Fund (QNRF), under project NPRP 5-401-2-161.
}

fading and frequency selective channels, respectively. In [6], the authors studied the design of precoders for multiple antenna systems to achieve various information and energy transmission tradeoffs. In [7], the authors proposed power allocation schemes for maximizing the energy efficiency of systems with concurrent information and power transfer. Yet, the results in [4]-[7] are obtained by assuming single layer transmission which does not capture the properties of multilayer transmission in multimedia applications.

Layered transmission is a promising approach for achieving a better resource utilization in multimedia applications and has been implemented in different multimedia standards such as JPEG2000 and H.264/SVC [8], [9]. Thereby, for instance, a video source signal is encoded into multiple layers with different source coding rates, i.e., a base layer and several enhancement layers. In particular, the base layer can be decoded independently from the other layers and the embedded information provides a basic video quality; the enhancement layers can only be decoded successively together with the base layer and they further refine the quality of the video information encoded in the base layer. As a result, the structure of layered transmission requires unequal error protection which introduces a paradigm shift in resource allocation algorithm design. On the other hand, a large amount of work has been recently devoted to physical (PHY) layer security [10]-[13], as a complement to traditional cryptographic encryption adopted in the application layer. Specifically, PHY layer security guarantees perfectly secure communication by exploiting the physical characteristics of the wireless communication channel. In [10], Wyner showed that when the legitimate receiver enjoys a better channel quality than the eavesdropper, the transmitter can deliver perfectly secure messages to the legitimate receiver at a non-zero data rate. In [11] and [12], artificial noise generation was proposed for providing secure communication in fast fading and slow fading channels, respectively. In [13], beamforming design was proposed to minimize the total transmit power of the system with simultaneous energy and secure information transfer. However, single layer transmission was adopted and the receivers in [10]-[13] were assumed to be powered by perpetual energy sources which may not be valid for power-constrained portable devices requiring multimedia services. Furthermore, an efficient power allocation scheme for secure layered transmission systems with energy harvesting receivers has not been reported in the literature [1]-[13].

In this paper, we address the above issues and study the power allocation algorithm design for multicast secure layered transmission systems with simultaneous wireless information and power transfer. The algorithm design is formulated as a non-convex optimization problem. To circumvent the problem intractability, we reformulate the optimization problem by introducing a convex tractable deterministic constraint. Subsequently, a semidefinite programming relaxation (SDR) based power allocation algorithm is proposed to obtain an upper bound solution for the reformulated problem. Besides, the upper 


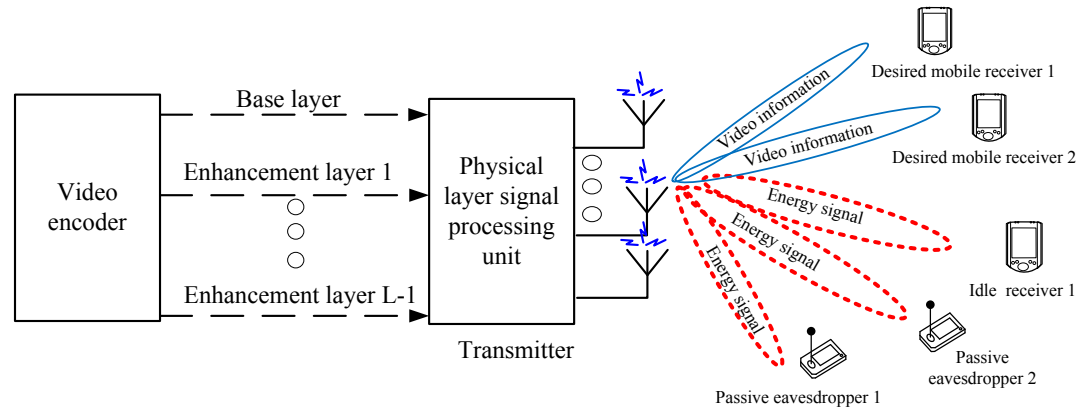

Fig. 1. A single group multicast communication system with layered transmission for $K=3$ mobile receivers (two active and one idle) and $J=2$ passive eavesdroppers.

bound solution is used as a building block for the design of two suboptimal schemes which are shown to achieve excellent system performance.

\section{SySTEM MODEL}

In this section, we present the adopted system model for secure layered video transmission.

\section{A. Channel Model}

We consider a single group multicast downlink communication which comprises a transmitter with $N_{\mathrm{T}}$ transmit antennas, $J$ single antenna passive eavesdroppers, and $K$ single antenna video receivers, cf. Figure 1. In every time instant, the transmitter conveys video information to a given set of multicast video service subscribers (receivers) requiring the same video information while the remaining receivers are idle. The eavesdroppers are assumed to be passive and silent to hide their existences. We focus on a time division duplexing system with a frequency flat slow time varying fading channel. The downlink channel gains of all legitimate receivers can be accurately obtained at the transmitter via measuring the uplink pilot sequences in the handshaking signals by exploiting channel reciprocity. The downlink received signals at desired video receiver $k \in\{1, \ldots, K\}$ and passive eavesdropper $j \in\{1, \ldots, J\}$ are given by, respectively,

$$
\begin{aligned}
y_{k} & =\mathbf{h}_{k}^{H} \mathbf{x}+z_{\mathrm{s}, k} \\
y_{\mathrm{PE}, j} & =\mathbf{g}_{j}^{H} \mathbf{x}+z_{\mathrm{e}, j},
\end{aligned}
$$

where $\mathbf{x} \in \mathbb{C}^{N_{\mathrm{T}} \times 1}$ denotes the transmitted symbol vector and $\mathbb{C}^{N \times M}$ denotes the space of $N \times M$ matrices with complex entries. $\mathbf{h}_{k}^{H} \in \mathbb{C}^{1 \times N_{\mathrm{T}}}$ is the channel vector between the transmitter and legitimate receiver $k$ and $\mathbf{g}_{j}^{H} \in \mathbb{C}^{1 \times N_{\mathrm{T}}}$ is the channel vector between the transmitter and passive eavesdropper $j$. $(\cdot)^{H}$ denotes the conjugate transpose of a matrix. $z_{\mathrm{s}, k}$ and $z_{\mathrm{e}, j}$ denote the joint effect of thermal noise and signal processing noise at desired video receiver $k$ and passive eavesdropper $j$, respectively. They are modeled as additive white Gaussian noises with zero mean and variance $\sigma_{\mathrm{s}}^{2}$.

\section{B. Information Decoding and Energy Harvesting Receiver}

We assume that each video receiver has the ability to harvest energy and to decode the modulated information 2 from the received radio signal. However, in practice, the signal used for decoding of the modulated information cannot be reused for harvesting energy due to hardware limitations [6]. As a result, a video receiver can either decode the video information when it is active (being served by the transmitter) or harvest energy when it is idle, but not performing both simultaneously,

\footnotetext{
${ }^{1}$ The results of this paper can be generalized to multigroup multicast networks at the expense of a more involved notation.

${ }^{2}$ Please refer to [5] for a hardware implementation of the energy harvesting circuitry.
}

cf. Figure 11 Besides, a rechargeable battery is used to store the harvested energy for future use. If the energy storage of the battery is full, the energy harvesting process will stop to prevent the battery from being overcharged. To facilitate energy harvesting at the idle receivers, the transmitter can increase the transmit power of the video information signal. However, this makes the video information more vulnerable to eavesdropping due to the high transmit power. As a result, a novel power allocation algorithm design is needed for promoting simultaneous power and information transfer while ensuring robustness against passive eavesdropping.

\section{Video Encoding and Energy Signal Generation}

A layered video encoding approach is adopted to encode the video information. Specifically, the video source signal is encoded into $L$ layers at the transmitter and the data rate of each layer is fixed, cf. H.264/SVC [14], [15]. The video information can be represented as $\mathbf{S}=\left[s_{1}, s_{2}, \ldots, s_{L}\right], s_{l} \in$ $\mathbb{C}, \forall l \in\{1, \ldots, L\}$, where $s_{l}$ denotes the video information of layer $l$. The $L$ layers include one base layer which contains the most essential information of the video. The base layer can be decoded independently without utilizing information from the other layers and the embedded information guarantees a minimum video quality. The remaining $L-1$ layers are enhancement layers which are used to successively refine the decoded lower layers. However, the information contained in a given enhancement layer cannot be decoded if there is a failure in decoding any of the lower layers.

On the other hand, an energy signal is transmitted along with the information signals to degrade the quality of the channels of the eavesdroppers and to facilitate energy harvesting at the idle receivers. Hence, the transmitter adopts the transmit symbol vector $\mathbf{x}$ as

$$
\mathbf{x}=\underbrace{\sum_{l=1}^{L} \mathbf{w}_{l} s_{l}}_{L \text {-layer video signal }}+\underbrace{\mathbf{w}_{\mathrm{E}}}_{\text {energy signal }},
$$

where $\mathbf{w}_{l} \in \mathbb{C}^{N_{\mathrm{T}} \times 1}$ is the beamforming vector for the video information in layer $l$ intended for the desired receivers. We note that superposition coding is used to superimpose the $L$ video information layers. $\mathbf{w}_{\mathrm{E}} \in \mathbb{C}^{N_{\mathrm{T}} \times 1}$ is the energy signal vector generated to cause interference to the passive eavesdroppers. In particular, $\mathbf{w}_{\mathrm{E}}$ is modeled as a complex Gaussian pseudo-random vector represented as $\mathbf{w}_{\mathrm{E}} \sim \mathcal{C N}\left(\mathbf{0}, \mathbf{W}_{\mathrm{E}}\right)$. $\mathbf{W}_{\mathrm{E}}$ denotes the covariance matrix of the energy signal, i.e., $\mathbf{W}_{\mathrm{E}} \in \mathbb{H}^{N_{\mathrm{T}}}$ and $\mathbf{W}_{\mathrm{E}} \succeq \mathbf{0}$. Here, $\mathbf{W}_{\mathrm{E}} \succeq \mathbf{0}$ indicates that $\mathbf{W}_{\mathrm{E}}$ is a positive semidefinite Hermitian matrix and $\mathbb{H}^{N}$ represents the set of all $N$-by- $N$ complex Hermitian matrices. Besides, the Gaussian pseudo-random energy signal is known at the legitimate receivers but is unavailable at the passive eavesdroppers. The transmitter regularly changes the seeds of the random sequence generator used for generating the energy 
signal sequences to prevent the sequence from being cracked by the passive eavesdroppers. Besides, the seeds information used at the transmitter can be delivered to the desired receivers securely by exploiting e.g. the reciprocity of the channels between the transmitter and the desired receivers [16].

\section{Power Allocation Algorithm Design}

In this section, we present the adopted performance metrics and the problem formulation.

\section{A. Channel Capacity}

We assume that perfect channel state information is available at the video receivers. Besides, successive interference cancellation [17] is performed at the receivers decoding the video information. Since the energy signal sequence is known at the legitimate receivers, the energy signal, $\mathbf{w}_{\mathrm{E}}$, is first removed from the received signal before video information decoding starts. Then, the receivers decode and cancel the lower video information layers successively before decoding the higher layers. Therefore, the instantaneous channel capacity between the transmitter and legitimate mobile video receiver $k \in\{1, \ldots, K\}$ in layer $l \in\{1, \ldots, L\}$ is given by

$$
\begin{aligned}
C_{l, k} & =\log _{2}\left(1+\Gamma_{l, k}\right) \text { and } \\
\Gamma_{l, k} & =\frac{\left|\mathbf{h}_{k}^{H} \mathbf{w}_{l}\right|^{2}}{\sum_{t=l+1}^{L}\left|\mathbf{h}_{k}^{H} \mathbf{w}_{t}\right|^{2}+\sigma_{\mathrm{s}}^{2}},
\end{aligned}
$$

where $\Gamma_{l, k}$ is the received signal-to-interference-plus-noise ratio (SINR) of layer $l$ at receiver $k$, and $|\cdot|$ denotes the absolute value of a complex scalar.

On the other hand, the channel state information of the passive eavesdroppers is not available at the transmitter. As a result, we design the power allocation algorithm for the worst case scenario. In particular, we assume that eavesdropper $j$ is close to the transmitter and is located at the reference distance of the path loss model. Then, an upper bound on the capacity of layer $l$ between the transmitter and passive eavesdropper $j \in\{1, \ldots, J\}$ under this worst case scenario is given by [11], [12]

$$
\begin{aligned}
C_{\mathrm{PE}_{l, j}} & =\log _{2}\left(1+\Gamma_{\mathrm{PE}_{l, j}}\right) \text { and } \\
\Gamma_{\mathrm{PE}_{l, j}} & =\frac{\mathbf{w}_{l}^{H} \mathbf{G}_{j} \mathbf{w}_{l}}{\sum_{t=l+1}^{L}\left|\mathbf{g}_{k}^{H} \mathbf{w}_{t}\right|^{2}+\operatorname{Tr}\left(\mathbf{G}_{j} \mathbf{W}_{\mathrm{E}}\right)+\sigma_{\mathrm{s}}^{2}} \\
& \stackrel{(a)}{\leq} \frac{\mathbf{w}_{l}^{H} \tilde{\mathbf{G}}_{j} \mathbf{w}_{l}}{\sum_{t=l+1}^{L} \mathbf{w}_{t}^{H} \tilde{\mathbf{G}}_{j} \mathbf{w}_{t}+\operatorname{Tr}\left(\tilde{\mathbf{G}}_{j} \mathbf{W}_{\mathrm{E}}\right)+\tilde{\sigma}_{\mathrm{s}, j}^{2}},
\end{aligned}
$$

where $\operatorname{Tr}(\cdot)$ denotes the trace of a matrix, $\mathbf{G}_{j}=\mathbf{g}_{j} \mathbf{g}_{j}^{H}$, $\tilde{\sigma}_{\mathrm{s}, j}^{2}=\frac{\sigma_{\mathrm{s}}^{2}}{\mathcal{E}\left\{\left\|\mathrm{g}_{j}^{\text {Ref }}\right\|^{2}\right\}}$, and $\mathbf{g}_{j}^{\text {Ref }}$ contains the channel coefficients of the eavesdropper at the reference distance. Here, $\|\cdot\|$ and $\mathcal{E}\{\cdot\}$ represent the Euclidean vector norm and statistical expectation, respectively. We note that the elements of $\mathbf{g}_{j}^{\text {Ref }}$ and $\mathbf{g}_{j}$ capture the joint effect of small scale fading and shadowing in the same manner. Yet, the path loss coefficient contained in $\mathbf{g}_{j}^{\text {Ref }}$ is calculated at the reference distance which results in $\left\|\mathbf{g}_{j}^{\text {Ref }}\right\| \geq\left\|\mathbf{g}_{j}\right\|$. Since $\mathcal{E}\left\{\left\|\mathbf{g}_{1}^{\operatorname{Ref}}\right\|^{2}\right\}=\ldots=\mathcal{E}\left\{\left\|\mathbf{g}_{j}^{\operatorname{Ref}}\right\|^{2}\right\}=\ldots=\mathcal{E}\left\{\left\|\mathbf{g}_{J}^{\text {Ref }}\right\|^{2}\right\}$, we replace $\tilde{\sigma}_{\mathrm{s}, j}^{2}$ by $\tilde{\sigma}_{\mathrm{s}}^{2}$ without loss of generality. Besides, $\tilde{\mathbf{G}}_{j}=\tilde{\mathbf{g}}_{j} \tilde{\mathbf{g}}_{j}^{H}=\frac{\mathbf{g}_{j}^{\text {Ref }}\left(\mathbf{g}_{j}^{\text {Ref }}\right)^{H}}{\mathcal{E}\left\{\left\|\mathbf{g}_{j}^{\text {Ref }}\right\|^{2}\right\}}$ in $(a)$ is a normalized matrix with $\left\|\tilde{\mathbf{g}}_{j}\right\|=1$. Furthermore, the passive eavesdroppers are unable to perform interference cancellation to remove $\operatorname{Tr}\left(\mathbf{G}_{j} \mathbf{W}_{\mathrm{E}}\right)$ since the energy signal $\mathbf{w}_{\mathrm{E}}$ is only known at the legitimate receivers. With a slight abuse of notation, we reuse variable $C_{\mathrm{PE}_{l, j}}$ to denote the upper bound on the capacity of layer $l$ at passive eavesdropper $j$ by replacing the $\operatorname{SINR} \Gamma_{\mathrm{PE}_{l, j}}$ in (6) with its upper bound in (7). Thus, the maximum secrecy capacity of layer $l$ between the transmitter and the desired active receivers is given by [11]

$$
C_{\mathrm{sec}_{l}}=\left[\min _{k \in \mathcal{A}} C_{l, k}-\max _{j \in\{1, \ldots, J\}} C_{\mathrm{PE}_{l, j}}\right]^{+},
$$

where $[x]^{+}=\max \{0, x\}$ and $\mathcal{A}$ denotes the set of active receivers which require video information.

\section{B. Optimization Problem Formulation}

The optimal power allocation policy $\left\{\mathbf{w}_{l}^{*}, \mathbf{W}_{\mathrm{E}}^{*}\right\}$ can be obtained by solving:

$$
\begin{gathered}
\underset{\mathbf{W}_{\mathrm{E}} \in \mathbb{H}^{N_{\mathrm{T}}, \mathbf{w}_{l}}}{\operatorname{minimize}} \sum_{l=1}^{L}\left\|\mathbf{w}_{l}\right\|^{2}+\operatorname{Tr}\left(\mathbf{W}_{\mathrm{E}}\right) \\
\text { s.t. C1: } \frac{\left|\mathbf{h}_{k}^{H} \mathbf{w}_{l}\right|^{2}}{\sum_{t=l+1}^{L}\left|\mathbf{h}_{k}^{H} \mathbf{w}_{t}\right|^{2}+\sigma_{\mathrm{s}}^{2}} \geq \Gamma_{\mathrm{req}_{l}}, \forall k \in \mathcal{P}, \forall l, \\
\mathrm{C} 2: \frac{\left|\mathbf{h}_{k}^{H} \mathbf{w}_{1}\right|^{2}}{\sum_{t=2}^{L}\left|\mathbf{h}_{k}^{H} \mathbf{w}_{t}\right|^{2}+\sigma_{\mathrm{s}}^{2}} \geq \Gamma_{\mathrm{req}_{1}}, \forall k \in \mathcal{B}, \\
\mathrm{C} 3: \operatorname{Pr}_{j \in\{1, \ldots, J\}}\left\{\Gamma_{\left.\mathrm{PE}_{1, j}\right\}} \max _{\mathrm{tol}_{1}}\right) \geq \kappa, \\
\mathrm{C} 4: \sum_{l=1}^{L}\left|\mathbf{h}_{k}^{H} \mathbf{w}_{l}\right|^{2}+\operatorname{Tr}\left(\mathbf{H}_{k} \mathbf{W}_{\mathrm{E}}\right) \geq \frac{P_{\min _{k}}}{\eta_{k}}, \forall k \in \mathcal{I}, \\
\mathrm{C} 5: \mathbf{W}_{\mathrm{E}} \succeq \mathbf{0},
\end{gathered}
$$

where $\mathbf{H}_{k}=\mathbf{h}_{k} \mathbf{h}_{k}^{H}$. $\Gamma_{\mathrm{req}_{l}}$ in $\mathrm{C} 1$ is the minimum required SINR for decoding layer $l$ at receiver $k$ and $\mathcal{P}$ is the set of receivers which subscribe the premium video service. In particular, the transmitter is required to guarantee the quality of service (QoS) (i.e., SINR) of each layer for the premium video service. $\mathcal{B}$ in $\mathrm{C} 2$ denotes the set of receivers which subscribe the basic video service. Constraint $\mathrm{C} 2$ indicates that the transmitter only guarantees a minimum required SINR for the first layer which provides the basic video quality. In $\mathrm{C} 3, \Gamma_{\mathrm{tol}_{1}}$ denotes the maximum receive SINR tolerance in layer 1 for decoding layer 1 successfully at passive eavesdropper $j$. In particular, the maximum SINR among all passive eavesdroppers is required to be smaller than $\Gamma_{\text {tol }_{1}}$ with at least probability $\kappa$. Since layered coding is employed for video information encoding, it is sufficient to protect the first video information layer against passive eavesdropping. If an eavesdropper is unable to decode layer 1 , then he/she will also not be able to decode layer $l \geq 2$. Besides, although the number of eavesdroppers $J$ is not known at the transmitter, $J$ in $\mathrm{C} 3$ represents the maximum tolerable number of passive eavesdroppers that the transmitter can handle. Furthermore, we do not maximize the secrecy capacity of video delivery in this paper as it does not necessarily lead to a power efficient power allocation. Yet, the problem formulation in (9) guarantees a minimum secrecy capacity of layer 1, i.e., $C_{\text {sec }_{1}} \geq \log _{2}\left(1+\Gamma_{\text {req }_{1}}\right)-\log _{2}\left(1+\Gamma_{\text {tol }_{1}}\right)$, with probability $\kappa$. Moreover, $\mathcal{I}$ in $\mathrm{C} 4$ represents the set of idle receivers and $P_{\min _{k}}$ denotes the minimum required power harvested at idle receiver $k .0<\eta_{k} \leq 1$ is a constant which denotes the efficiency of the energy harvesting circuit for converting the received radio signal to electrical energy. We note that sets $\mathcal{P}, \mathcal{I}, \mathcal{B}$ are mutually exclusive and $\mathcal{P}, \mathcal{B} \in \mathcal{A}$. C5 and $\mathbf{W}_{\mathrm{E}} \in \mathbb{H}^{N_{\mathrm{T}}}$ are imposed such that $\mathbf{W}_{\mathrm{E}}$ satisfies the requirements on the covariance matrix of the energy signal. We also point out that the benefits of energy signal generation are two-fold. First, the energy signal is used to compromise the channels of the passive eavesdroppers for providing communication security, cf. C3 and (7). Second, 
it acts as a energy source for the idle receivers for energy harvesting, cf. $\mathrm{C} 4$.

\section{Solution of the Optimization Problem}

The problem in (9) is a non-convex optimization problem. In order to obtain a tractable power allocation algorithm, we first handle constraints $\mathrm{C} 1, \mathrm{C} 2$, and $\mathrm{C} 4$ by transforming the problem into an equivalent. Subsequently, we reformulate the considered problem by replacing the probabilistic constraint $\mathrm{C} 3$ with a convex deterministic constraint. Then, an semidefinite programming relaxation (SDR) approach is adopted to obtain an upper bound solution for the reformulated problem. Finally, we propose two suboptimal power allocation schemes which achieve excellent system performances.

\section{A. Semidefinite Programming Relaxation}

First, we rewrite problem (9) in an equivalent form via SDP:

$$
\begin{gathered}
\underset{\mathbf{W}_{l}, \mathbf{W}_{\mathrm{E}} \in \mathbb{H}^{N_{\mathrm{T}}}}{\operatorname{minimize}} \sum_{l=1}^{L} \operatorname{Tr}\left(\mathbf{W}_{l}\right)+\operatorname{Tr}\left(\mathbf{W}_{\mathrm{E}}\right) \\
\text { s.t. C1: } \frac{\operatorname{Tr}\left(\mathbf{H}_{k} \mathbf{W}_{l}\right)}{\sum_{t=l+1}^{L} \operatorname{Tr}\left(\mathbf{H}_{k} \mathbf{W}_{t}\right)+\sigma_{\mathrm{s}}^{2}} \geq \Gamma_{\mathrm{req}_{l}}, \forall k \in \mathcal{P}, \forall l, \\
\text { C2: } \frac{\operatorname{Tr}\left(\mathbf{H}_{k} \mathbf{W}_{1}\right)}{\sum_{t=2}^{L} \operatorname{Tr}\left(\mathbf{H}_{k} \mathbf{W}_{t}\right)+\sigma_{\mathrm{s}}^{2}} \geq \Gamma_{\mathrm{req}_{1}}, \forall k \in \mathcal{B}, \\
\text { C3: } \operatorname{Pr}\left(\max _{j \in\{1, \ldots, J\}}\left\{\Gamma_{\left.\mathrm{PE}_{1, j}\right\}}\right\} \Gamma_{\mathrm{tol}_{1}}\right) \geq \kappa, \\
\text { C4: } \operatorname{Tr}\left(\mathbf{H}_{k}\left(\mathbf{W}_{\mathrm{E}}+\sum_{l=1}^{L} \mathbf{W}_{l}\right)\right) \geq \frac{P_{\min _{k}}}{\eta_{k}}, \forall k \in \mathcal{I}, \\
\text { C5: } \mathbf{W}_{\mathrm{E}} \succeq \mathbf{0}, \quad \mathrm{C} 6: \mathbf{W}_{l} \succeq \mathbf{0}, \forall l \in\{1, \ldots, L\}, \\
\text { C7: } \operatorname{Rank}\left(\mathbf{W}_{l}\right)=1, \forall l \in\{1, \ldots, L\},
\end{gathered}
$$

where $\mathbf{W}_{l}=\mathbf{w}_{l} \mathbf{w}_{l}^{H}$ and $\operatorname{Rank}(\cdot)$ in $\mathrm{C} 7$ denotes the rank of an input matrix. We note that $\mathbf{W}_{l} \succeq \mathbf{0}, \forall l \in\{1, \ldots, L\}$, $\mathbf{W}_{l} \in \mathbb{H}^{N_{\mathrm{T}}}, \forall l$, and $\operatorname{Rank}\left(\mathbf{W}_{l}\right)=1, \forall \bar{l}$, in (10) are imposed to guarantee that $\mathbf{W}_{l}=\mathbf{w}_{l} \mathbf{w}_{l}^{H}$ holds after optimizing $\mathbf{W}_{l}$. The transformed problem above is still non-convex due to the probabilistic constraint $\mathrm{C} 3$ and the rank constraint in $\mathrm{C} 7$. To overcome this problem, we introduce the following lemma for reformulating the considered problem:

Lemma 1: Assuming the normalized upper bound channel gain vectors of the passive eavesdroppers can be modeled as independent and identical distributed (i.i.d.) Rayleigh random variables, $\tilde{\mathbf{g}}_{j}$, the following constraint implication holds:

$$
\begin{aligned}
& \overline{\mathrm{C} 3}: \Phi_{N_{\mathrm{T}}}^{-1}\left(1-\kappa^{1 / J}\right) \Gamma_{\mathrm{tol}_{1}} \tilde{\sigma}_{\mathrm{s}}^{2} \geq \lambda_{\max }(\mathbf{Q}) \\
\Rightarrow \mathrm{C} 3: & \operatorname{Pr}\left(\max _{j \in\{1, \ldots, J\}}\left\{\Gamma_{\mathrm{PE}_{1, j}}\right\} \leq \Gamma_{\mathrm{tol}_{1}}\right) \geq \kappa,
\end{aligned}
$$

where $\mathbf{Q}=\mathbf{W}_{1}-\Gamma_{\text {tol }_{1}}\left(\sum_{t=2}^{L} \mathbf{W}_{t}+\mathbf{W}_{\mathrm{E}}\right), \Phi_{N_{\mathrm{T}}}^{-1}(\cdot)$ denotes the inverse cumulative distribution function (c.d.f.) of an inverse central chi-square random variable with $2 N_{\mathrm{T}}$ degrees of freedom and $\lambda_{\max }(\cdot)$ denotes the maximum eigenvalue of a square input matrix.

Proof: Please refer to Appendix A.

As a result, we can replace the probabilistic constraint $\mathrm{C} 3$ with the constraint in 111 . Constraint $\overline{\mathrm{C} 3}$ is safe and tractable in the sense that: (i) a feasible solution point satisfying $\overline{\mathrm{C} 3}$ will also satisfy $\mathrm{C} 3$, and (ii) the new constraint $\overline{\mathrm{C} 3}$ is a convex function with respect to the optimization variables 3 .

\footnotetext{
${ }^{3}$ Although the maximum eigenvalue function is a non-smooth function, it is a convex function [18].
}

Thus, replacing probabilistic constraint $\mathrm{C} 3$ with $\overline{\mathrm{C} 3}$ in (11), we obtain the following reformulated optimization problem:

$$
\begin{gathered}
\underset{\mathbf{W}_{l}, \mathbf{W}_{\mathrm{E}} \in \mathbb{H}^{N_{\mathrm{T}}}}{\operatorname{minimize}} \sum_{l=1}^{L} \operatorname{Tr}\left(\mathbf{W}_{l}\right)+\operatorname{Tr}\left(\mathbf{W}_{\mathrm{E}}\right) \\
\text { s.t. } \quad \mathrm{C} 1, \mathrm{C} 2, \mathrm{C} 4, \mathrm{C} 5, \mathrm{C} 6, \mathrm{C} 7, \\
\left.\overline{\mathrm{C} 3}: \Gamma_{\mathrm{tol}_{1}} \tilde{\sigma}_{\mathrm{s}}^{2} \Phi_{N_{\mathrm{T}}}^{-1}\left(1-\kappa^{1 / J}\right) \geq \lambda_{\max }(\mathbf{Q})\right) .
\end{gathered}
$$

We note that the solution of the reformulated problem in 12 serves as a performance lower bound of (10) as a smaller feasible solution set is considered by replacing probabilistic constraint C3 with $\overline{\mathrm{C} 3}$. Now, C7: $\operatorname{Rank}\left(\mathbf{W}_{l}\right)=1, \forall l$, is the remaining obstacle in solving the reformulated problem in (12). In fact, if this constraint is removed from the problem formulation, the reformulated problem becomes a convex SDP which satisfies Slater's constraint qualification. Thus, the SDP relaxed version of the reformulated problem in 12 can be solved efficiently by off-the-shelf numerical solvers such as SeDuMi [19]. In particular, if the obtained solution admits a rank-one matrix $\mathbf{W}_{l}, \forall l$, then it is the optimal solution of the original problem in 12. Yet, the proposed constraint relaxation may not be tight with respect to the reformulated problem and the result of the relaxed problem serves as a performance upper bound for the reformulated problem. Therefore, it is important to study under what condition(s) the solution of the problem in (12) yields rank-one matrices $\mathbf{W}_{l}, \forall l$.

\section{B. Optimality Conditions}

We investigate some sufficient conditions for obtaining a rank-one solution $\mathbf{W}_{l}$ via the Karush-Kuhn-Tucker (KKT) conditions of problem (12). To this end, we express the Lagrangian function of (12) as

$$
\begin{aligned}
& \mathcal{L}\left(\mathbf{W}_{l}, \mathbf{W}_{\mathrm{E}}, \boldsymbol{\mu}, \boldsymbol{\beta}, \boldsymbol{\delta}, \phi, \mathbf{Y}_{l}, \mathbf{X}\right) \\
= & \sum_{l=1}^{L} \operatorname{Tr}\left(\mathbf{W}_{l}\left(\mathbf{I}_{N_{\mathrm{T}}}-\mathbf{Y}_{l}\right)\right)-\phi \Phi_{N_{\mathrm{T}}}^{-1}\left(1-\kappa^{1 / J}\right) \Gamma_{\mathrm{tol}_{1}} \tilde{\sigma}_{\mathrm{s}}^{2} \\
- & \operatorname{Tr}\left(\mathbf{W}_{\mathrm{E}} \mathbf{X}\right)+\sum_{k \in \mathcal{P}} \sum_{l=1}^{L} \mu_{l, k}\left(\frac{-\operatorname{Tr}\left(\mathbf{H}_{k} \mathbf{W}_{l}\right)}{\Gamma_{\mathrm{req}_{l}}}+\sum_{t=l+1}^{L} \operatorname{Tr}\left(\mathbf{H}_{k} \mathbf{W}_{l}\right)\right. \\
+ & \left.\sigma_{\mathrm{s}}^{2}\right)+\phi \lambda_{\max }\left(\mathbf{W}_{1}-\Gamma_{\mathrm{tol}_{1}}\left(\sum_{t=2}^{L} \mathbf{W}_{t}+\mathbf{W}_{\mathrm{E}}\right)\right)+\operatorname{Tr}\left(\mathbf{W}_{\mathrm{E}}\right) \\
+ & \sum_{k \in \mathcal{B}} \beta_{k}\left(-\frac{\operatorname{Tr}\left(\mathbf{H}_{k} \mathbf{W}_{1}\right)}{\Gamma_{\mathrm{req}_{1}}}+\sum_{j=2}^{L} \operatorname{Tr}\left(\mathbf{H}_{k} \mathbf{W}_{j}\right)+\sigma_{\mathrm{s}}^{2}\right) \\
+ & \sum_{k \in \mathcal{I}} \delta_{k}\left(\frac{P_{\min _{k}}}{\eta_{k}}-\operatorname{Tr}\left(\mathbf{H}_{k}\left(\mathbf{W}_{\mathrm{E}}+\sum_{l=1}^{L} \mathbf{W}_{l}\right)\right)\right)
\end{aligned}
$$

where $\mathbf{I}_{N_{\mathrm{T}}}$ is the $N_{\mathrm{T}} \times N_{\mathrm{T}}$ identity matrix. $\boldsymbol{\mu}$, with elements $\mu_{l, k} \geq 0, \forall l \in\{1, \ldots, L\}, \forall k \in \mathcal{P}$, is the Lagrange multiplier vector associated with the minimum required SINR in decoding layer $l$ for receiver $k$ subscribing premium video service in $\mathrm{C} 1$. $\boldsymbol{\beta}$, with elements $\beta_{k} \geq 0, \forall k \in \mathcal{B}$, is the minimum required SINR in decoding layer 1 for receiver $k$ requesting basic video service in $\mathrm{C} 2 . \phi$ is the Lagrange multiplier associated with the proposed constraint $\overline{\mathrm{C} 3}$ from Lemma 1 . $\delta$, with elements $\delta_{k} \geq 0, \forall k \in \mathcal{I}$, is the Lagrange multiplier vector for the minimum required power transfer to the idle receivers in $\mathrm{C} 4$. Matrices $\mathbf{X}, \mathbf{Y}_{l} \succeq \mathbf{0}$ are the Lagrange multipliers for the positive semidefinite constraints on matrices $\mathbf{W}_{\mathrm{E}}$ and $\mathbf{W}_{l}$ in C5 and C6, respectively. The resulting dual problem for the SDP relaxed problem is given by

$$
\underset{\substack{\boldsymbol{\mu}, \boldsymbol{\beta}, \boldsymbol{\delta}, \phi \succeq \mathbf{0} \\ \mathbf{Y}, \mathbf{X} \succ \mathbf{0}}}{\operatorname{maximizimizize}} \underset{\mathbf{W}_{l}, \mathbf{W}_{\mathrm{E}} \in \mathbb{H}^{N_{\mathrm{T}}}}{\operatorname{Lin}} \mathcal{L}\left(\mathbf{W}_{l}, \mathbf{W}_{\mathrm{E}}, \boldsymbol{\mu}, \boldsymbol{\beta}, \boldsymbol{\delta}, \phi, \mathbf{Y}_{l}, \mathbf{X}\right) \text {. }
$$


In the following theorem, we reveal the rank of $\mathbf{W}_{l}^{*}$ in the relaxed version of problem (12).

Theorem 1: In general, for $\Gamma_{\mathrm{req}_{l}}>0, \forall l$, the following rank inequality for $\mathbf{W}_{1}^{*}$ holds:

$$
\operatorname{Rank}\left(\mathbf{W}_{1}^{*}\right) \leq \min \left\{K, N_{\mathrm{T}}\right\}
$$

Moreover, for $\phi=0$, i.e., constraint $\overline{\mathrm{C} 3}$ is not active at the optimal solution, there exist $\mathbf{W}_{l}^{*}$ such that

$$
\begin{aligned}
& \sum_{l=1}^{L} \operatorname{Rank}^{2}\left(\mathbf{W}_{l}^{*}\right)+\operatorname{Rank}^{2}\left(\mathbf{W}_{\mathrm{E}}^{*}\right) \\
& \leq \quad \operatorname{card}(\mathcal{P}) L+\operatorname{card}(\mathcal{B})+\operatorname{card}(\mathcal{I})
\end{aligned}
$$

where $\operatorname{card}(\cdot)$ denotes the cardinality of a set.

Proof: Please refer to Appendix B.

By utilizing Theorem 1, we summarize some sufficient conditions for obtaining a rank-one solution via SDP relaxation in the following corollary.

Corollory 1: For $\Gamma_{\mathrm{req}_{l}}>0, \forall l$, and $\phi=0$, the SDP relaxation of (12) is tight (i.e., $\operatorname{Rank}\left(\mathbf{W}_{l}\right)=1, \forall l$,) if one of the following is satisfied:

i) $L$ is arbitrary, $K \leq 2: \operatorname{card}(\mathcal{P})=1$

ii) $L=1, K=3: \operatorname{card}(\mathcal{P})=2, \operatorname{card}(\mathcal{B})=1, \operatorname{card}(\mathcal{I})=0$

iii) $L=1, K=3: \operatorname{card}(\mathcal{P})=2, \operatorname{card}(\mathcal{B})=0, \operatorname{card}(\mathcal{I})=1$

We note that Corollary 1 provides only sufficient conditions for the optimality of SDP relaxation. In practice, the SDP relaxation can admit a rank-one solution even if the sufficient conditions stated in Corollary 1 are not satisfied.

In the following, two suboptimal power allocation schemes are designed based on the framework of convex optimization.

1) Suboptimal Power Allocation Scheme 1: The first proposed suboptimal power allocation scheme is a hybrid scheme based on the solution of the relaxed version of (12). In particular, we first solve (12) by SDP relaxation. If the solution admits rank-one $\mathbf{W}_{l}, \forall l$, then the global optimal solution of (12) is obtained. Otherwise, we construct a suboptimal solution set $\widetilde{\mathbf{W}}_{l}=\widetilde{\mathbf{w}}_{l} \widetilde{\mathbf{w}}_{l}^{H}$, where $\widetilde{\mathbf{w}}_{l}$ is the eigenvector corresponding to the maximum eigenvalue of matrix $\mathbf{W}_{l}$, where $\mathbf{W}_{l}$ is the solution of the SDP relaxed version of 12 with $\operatorname{Rank}\left(\mathbf{W}_{l}\right)>1$. Then, we define $L$ scaling constants $\alpha_{l}, \forall l \in\{1, \ldots, L\}$ and a new optimization problem:

$$
\begin{gathered}
\underset{\alpha_{l}, \mathbf{W}_{\mathrm{E}} \in \mathbb{H}^{N_{\mathrm{T}}}}{\operatorname{minimize}} \sum_{l=1}^{L} \alpha_{l} \operatorname{Tr}\left(\widetilde{\mathbf{W}}_{l}\right)+\operatorname{Tr}\left(\mathbf{W}_{\mathrm{E}}\right) \\
\text { s.t. } \mathrm{C} 1: \frac{\alpha_{l} \operatorname{Tr}\left(\mathbf{H}_{k} \widetilde{\mathbf{W}}_{l}\right)}{\operatorname{Tr}\left(\mathbf{H}_{k}\left(\mathbf{W}_{\mathrm{E}}+\sum_{t=l+1}^{L} \alpha_{t} \widetilde{\mathbf{W}}_{t}\right)\right)+\sigma_{\mathrm{s}}^{2}} \geq \Gamma_{\mathrm{req}_{l}}, \forall k \in \mathcal{P}, \forall l, \\
\mathrm{C} 2: \frac{\alpha_{1} \operatorname{Tr}\left(\mathbf{H}_{k} \mathbf{W}_{1}\right)}{\operatorname{Tr}\left(\mathbf{H}_{k}\left(\mathbf{W}_{\mathrm{E}}+\sum_{t=2}^{L} \alpha_{t} \widetilde{\mathbf{W}}_{t}\right)\right)+\sigma_{\mathrm{s}}^{2}} \geq \Gamma_{\mathrm{req}_{1}}, \forall k \in \mathcal{B}, \\
\overline{\mathrm{C} 3:} \Phi_{N_{\mathrm{T}}}^{-1}\left(1-\kappa^{1 / J}\right) \Gamma_{\mathrm{tol}_{1}} \tilde{\sigma}_{\mathrm{s}}^{2} \geq \lambda_{\max }\left(\tilde{\mathbf{Q}}_{)},\right. \\
\mathrm{C} 4: \operatorname{Tr}\left(\mathbf{H}_{k}\left(\mathbf{W}_{\mathrm{E}}+\sum_{l=1}^{L} \alpha_{l} \widetilde{\mathbf{W}} l\right)\right) \geq \frac{P_{\min _{k}}}{\eta_{k}}, \forall k \in \mathcal{I}, \\
\mathrm{C} 5: \mathbf{W}_{\mathrm{E}} \succeq \mathbf{0}, \quad \mathrm{C} 6: \alpha_{l} \geq 0, \forall l,
\end{gathered}
$$

where $\tilde{\mathbf{Q}}=\alpha_{1} \widetilde{\mathbf{W}}_{1}-\Gamma_{\mathrm{tol}_{1}}\left(\sum_{t=2}^{L} \alpha_{t} \widetilde{\mathbf{W}}_{t}+\mathbf{W}_{\mathrm{E}}\right)$. The problem formulation in 177 is convex with respect to the optimization variables. In particular, it serves as a suboptimal solution for (12).
2) Suboptimal Power Allocation Scheme 2: The second proposed suboptimal power allocation scheme is also a hybrid scheme. In particular, it is based on the solution of the relaxed version of (12) and the rank-one Gaussian randomization scheme [2]. Besides, a similar approach to solving the problem is adopted as in suboptimal power allocation scheme 1 , except for the choice of beamforming matrix $\widetilde{\mathbf{W}}_{l}$ when $\operatorname{Rank}\left(\mathbf{W}_{l}\right)>1$. Specifically, we calculate the eigenvalue decomposition of $\mathbf{W}_{l}=\mathbf{U}_{l} \boldsymbol{\Sigma}_{l} \mathbf{U}_{l}^{H}$, where $\mathbf{U}_{l}$ and $\boldsymbol{\Sigma}_{l}$ are an $N_{\mathrm{T}} \times N_{\mathrm{T}}$ unitary matrix and a diagonal matrix, respectively. Then, we adopt the suboptimal beamforming vector as $\widetilde{\mathbf{w}}_{l}=\mathbf{U}_{l} \boldsymbol{\Sigma}_{l}^{1 / 2} \mathbf{r}_{l}, \widetilde{\mathbf{W}}_{l}=\alpha_{l} \widetilde{\mathbf{w}}_{l} \widetilde{\mathbf{w}}_{l}^{H}$, where $\mathbf{r}_{l} \in \mathbb{C}^{N_{\mathrm{T}}}$ and $\mathbf{r}_{l} \sim \mathcal{C N}\left(\mathbf{0}, \mathbf{I}_{N_{\mathrm{T}}}\right)$. Subsequently, we follow the same approach as in (17) for optimizing $\left\{\alpha_{l}, \mathbf{W}_{\mathrm{E}}\right\}$ and obtain a suboptimal rank-one solution $\alpha_{l} \widetilde{\mathbf{W}}_{l}$.

\section{RESULTS}

In this section, we evaluate the system performance for the proposed power allocation schemes using simulations. We adopt the TGn path loss model [20] for indoor communication with a reference distance of 2 meters for the path loss model and a carrier center frequency of $470 \mathrm{MHz}$ [21]. There are $K$ legitimate video receivers uniformly distributed between the reference distance and the maximum service distance of 20 meters. The transmitter is equipped with $N_{\mathrm{T}}$ antennas and we assume a joint transmit and receive antenna gain of 10 $\mathrm{dBi}$ (isotropic). The multipath fading coefficients between the transmitter and legitimate video receivers are generated as i.i.d. Rician random variables with Rician factor $6 \mathrm{~dB}$. On the other hand, we assume that there are $J=4$ eavesdroppers eavesdropping the video information from outdoor 4 . Thus, the multipath fading coefficients between the transmitter and the $J$ passive eavesdroppers are modeled as Rayleigh random variables. The noise power and the RF energy conversion efficiency at the receivers are $\sigma_{\mathrm{s}}^{2}=-33 \mathrm{dBm}$ and $\eta_{k}=0.5, \forall k$, respectively. The video signal is encoded into 3 layers with minimum SINR requirements of $\Gamma_{\mathrm{req}_{1}}=6 \mathrm{~dB}, \Gamma_{\mathrm{req}_{2}}=9 \mathrm{~dB}$, and $\Gamma_{\mathrm{req}_{3}}=12$ $\mathrm{dB}$, respectively. The maximum tolerable SINR at eavesdropper $j$ is set to $\Gamma_{\text {tol }_{1}}=-10 \mathrm{~dB}$ and $\kappa=0.99$. In other words, the proposed power allocation schemes guarantee a minimum secrecy capacity of layer 1 video information of $C_{\mathrm{sec}_{1}} \geq 2.179$ bit/s/Hz with 0.99 probability. Unless specified otherwise, we assume that there are two idle receivers requiring minimum harvested powers of $P_{\min _{k}}=0 \mathrm{dBm}, \forall k \in \mathcal{I}$, and two video receivers requiring basic video service. The system performance is obtained by averaging over 50000 multipath fading and path loss realizations.

\section{A. Average Total Transmit Power}

Figure 2 depicts the average total transmit power versus the number of video receivers $K$ for different power allocation schemes and different numbers of transmit antennas $N_{\mathrm{T}}$. It can be observed that the average total transmit power of the proposed schemes is a monotonically non-decreasing function of the number of video receivers. This is because a higher transmit power is required for satisfying constraint $\mathrm{C} 1$ when there are more video receivers requesting the premium video service. Besides, the two proposed suboptimal schemes perform closely to the upper bound system performance achieved by SDP relaxation. In fact, the proposed suboptimal schemes 1 and 2 exploit the possibility of achieving the global optimal solution via SDP relaxation. On the other hand, the total transmit power decreases with an increasing number of transmit antennas. This

\footnotetext{
${ }^{4}$ Although the eavesdroppers are located outdoor, this location information is not known at the transmitter. The problem formulation in 9 assumes Rayleigh fading channels for passive eavesdroppers and considers the worst case scenario in 7 for providing secure communication.
} 


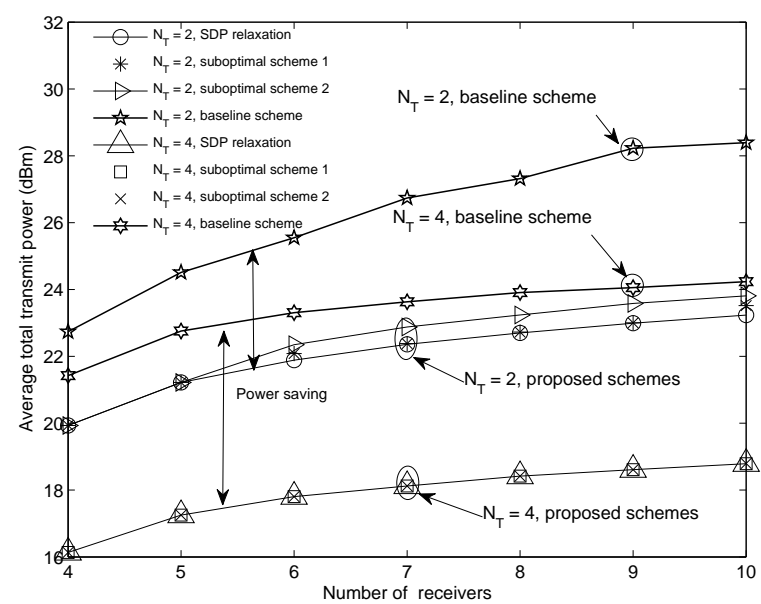

Fig. 2. Average total transmit power $(\mathrm{dBm})$ versus the number of receivers for different power allocation schemes and different numbers of transmit antennas $N_{\mathrm{T}}$. The double-sided arrows indicate the power savings achieved by the proposed schemes compared to the baseline scheme.

is attributed to the fact that the degrees of freedom for power allocation increase with increasing number of transmit antennas which facilities the video information transmission.

For comparison, Figure 2 also contains the average total transmit power of a baseline power allocation scheme. The baseline scheme is a hybrid scheme. In particular, we adopt maximum ratio transmission (MRT) [3] for delivering the video information of each layer with respect to the active receiver with the highest channel gain, i.e., $\max _{k \in \mathcal{A}}\left\|\mathbf{h}_{k}\right\|^{2}$. As for the energy signal, we adopt an isotropic radiation pattern for $\mathbf{W}_{\mathrm{E}}$. Then, we optimize both the power allocated to $\mathbf{W}_{\mathrm{E}}$ and the MRT beamforming vector for minimizing the total transmit power subject to the same constraints as in (12). Although the baseline scheme requires a lower computational complexity than the other schemes, it can be observed from Figure 2 that the baseline scheme has the worst performance among all considered schemes. This is because the transmitter is unable to fully exploit the degrees of freedom in power allocation when $\mathbf{W}_{\mathrm{E}}$ is radiated isotropically and $\mathbf{W}_{l}$ is fixed.

\section{B. Average Harvested Power}

Figure 3 shows the average harvested power versus the number of receivers for different power allocation schemes. The average harvested power increases with the number of receivers. In fact, a higher transmit power is required for fulfilling the SINR requirements in $\mathrm{C} 1$ as the number of receivers increase which results in a higher energy level in the RF. Besides, the two proposed suboptimal power allocation schemes exhibit a similar performance as the SDR upper bound solution and are able to guarantee the minimum required harvesting power of the idle video receivers. On the other hand, as expected, the idle receivers are able to harvest more energy for the baseline scheme than for the proposed schemes since for the baseline scheme, the transmitter has to transmit an exceedingly large amount of power in the RF to satisfy all the QoS requirements which benefits energy harvesting.

\section{CONCLUSIONS}

In this paper, we studied the power allocation algorithm design for secure layered multicast video transmission systems with simultaneous information and power transfer. The algorithm design was formulated as a non-convex optimization problem by taking into account energy signal generation to facilitate secure communication and efficient energy harvesting. Due to the intractability of the problem, the considered problem

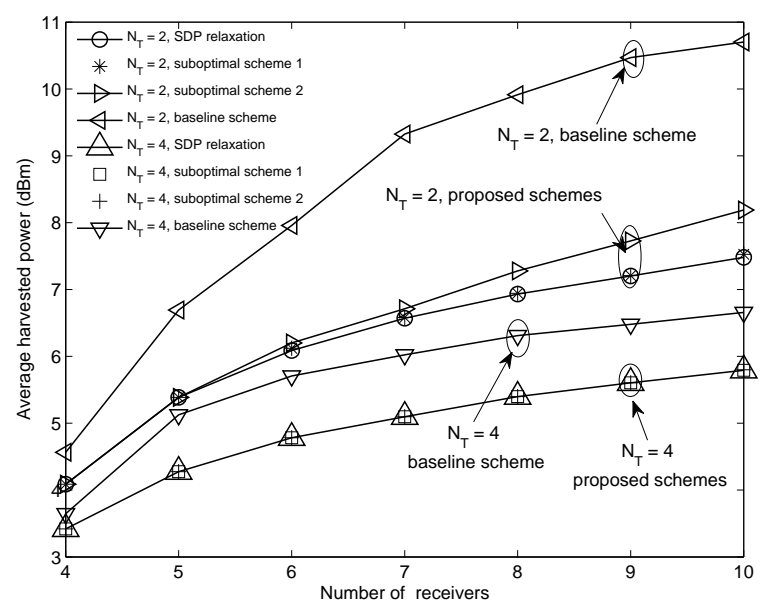

Fig. 3. Average total harvested power $(\mathrm{dBm})$ versus the number of receivers for different power allocation schemes and different numbers of transmit antennas $N_{\mathrm{T}}$.

was reformulated by introducing a convex deterministic constraint. Subsequently, an SDP relaxation based power allocation algorithm was proposed to solve the non-convex optimization problem which resulted in an upper bound solution of the reformulated problem. Besides, two suboptimal power allocation schemes were designed exploiting the structure of the upper bound solution. Simulation results demonstrated the excellent performance of the proposed suboptimal schemes. In our future work, we will study the tightness of the proposed deterministic constraint and the impact of imperfect knowledge of the channel state information of the video receivers.

\section{APPENDIX}

\section{A. Proof of Lemma 1}

By exploiting the independence of the $J$ passive eavesdroppers channels and after some mathematical manipulation, the left hand side of constraint $\mathrm{C} 3$ in (9) can be equivalently written as

$$
\operatorname{Pr}\left(\Gamma_{\mathrm{tol}_{1}} \tilde{\sigma}_{\mathrm{s}}^{2} \geq \operatorname{Tr}(\tilde{\mathbf{G}} \mathbf{Q})\right) \geq \kappa^{1 / J} .
$$

Note that we drop the index $j$ of the passive eavesdropper channels $\tilde{\mathbf{G}}_{j}$ in $(18)$ since the equivalent channels of the passive eavesdroppers are modeled as i.i.d. random variables. On the other hand, the probability distribution in (18) may not be a convex function and thus we focus on a smaller convex feasible solution set which provides a tractable solution. First, by utilizing the trace inequality in in [22, Lemma II.1], we obtain

$$
\operatorname{Tr}(\tilde{\mathbf{G}} \mathbf{Q}) \leq \operatorname{Tr}(\tilde{\mathbf{G}}) \lambda_{\max }(\mathbf{Q}) .
$$

Then, by combining (18) and (19), the following implication holds:

$$
\begin{aligned}
& \Phi_{N_{\mathrm{T}}}^{-1}\left(1-\kappa^{1 / J}\right) \Gamma_{\mathrm{tol}_{1}} \tilde{\sigma}_{\mathrm{s}}^{2} \leq \lambda_{\max }(\mathbf{Q}) \\
\Longleftrightarrow & \operatorname{Pr}\left(\frac{\lambda_{\max }(\mathbf{Q})}{\Gamma_{\mathrm{tol}_{1}} \tilde{\sigma}_{\mathrm{S}}^{2}} \leq \frac{1}{\operatorname{Tr}(\tilde{\mathbf{G}})}\right) \geq \kappa^{1 / J} \Rightarrow 18 \Rightarrow \mathrm{C} 3,
\end{aligned}
$$

where $\Phi_{N_{T}}^{-1}(\cdot)$ denotes the inverse cumulative distribution function (c.d.f.) of an inverse central chi-square random variable with $2 N_{\mathrm{T}}$ degrees of freedom. We note that random variable $\tilde{\mathbf{G}}$ is decoupled from the optimization variables, cf. (19). Thus, the implication in 20 is applicable to any continuous channel distribution by replacing $\Phi_{N_{\mathrm{T}}}^{-1}(\cdot)$ with the inverse c.d.f. of the corresponding distribution. On the other hand, the inverse c.d.f. can be obtained via a look-up table or by using the bisection method for practical implementation. 


\section{B. Proof of Theorem 1}

In the following, we study the rank of the optimal $\mathbf{W}_{l}^{*}$ via focusing on the corresponding KKT conditions:

$$
\begin{aligned}
\mathbf{Y}_{l}^{*} & \succeq \mathbf{0}, \quad \beta_{k}^{*}, \delta_{n}^{*}, \mu_{l, k}^{*}, \phi^{*} \geq 0, \forall l, n, k, \\
\mathbf{Y}_{l}^{*} \mathbf{W}_{l}^{*} & =\mathbf{0}, \forall l, \\
\mathbf{0} & \in \partial_{\mathbf{W}_{l}} \mathcal{L}(\boldsymbol{\Xi}), \forall l,
\end{aligned}
$$

where $\boldsymbol{\Xi}=\left\{\mathbf{W}_{l}, \mathbf{W}_{\mathrm{E}}, \boldsymbol{\mu}, \boldsymbol{\beta}, \boldsymbol{\delta}, \phi, \mathbf{Y}_{l}, \mathbf{X}\right\} \quad$ and $\mathbf{Y}_{l}^{*}, \beta_{k}^{*}, \delta_{n}^{*}, \mu_{l, k}^{*}, \phi^{*}$ are the optimal Lagrange multipliers for (14). Equation (22) is the complementary slackness condition. We note that $\mathbf{0} \in \partial_{\mathbf{W}_{l}} \mathcal{L}(\boldsymbol{\Xi})$ in (23) represents a set of subdifferentials with respect to $\mathbf{W}_{l}$ due to the non-differentiability of the maximum eigenvalue function in $\overline{\mathrm{C} 3}$. To obtain an expression for 23, we first define the sub-differential of the maximum eigenvalue function. The sub-differential of $\lambda_{\max }(\mathbf{Q})$ with respect to $\mathbf{Q}$ can be expressed as [23]

$$
\begin{aligned}
& \partial_{\mathbf{Q}} \lambda_{\max }(\mathbf{Q}) \\
= & \operatorname{convhull}\left\{\mathbf{z}_{n} \mathbf{z}_{n}^{H} \mid\left\|\mathbf{z}_{n}\right\|^{2}=1, \mathbf{Q} \mathbf{z}_{n}=\lambda_{\max }(\mathbf{Q}) \mathbf{z}_{n}\right\},
\end{aligned}
$$

where $\mathbf{z}_{n}$ is the $n$-th eigenvector of matrix $\mathbf{Q}$. Indeed, the subdifferential of the maximum eigenvalue function is a convex hull of the sub-gradients which satisfy 24). Thus, without loss of generally, we can represent the element in the set which satisfies $\mathbf{0}=\partial_{\mathbf{W}_{1}} \mathcal{L}(\boldsymbol{\Xi})$ as

$$
\sum_{n=1}^{N_{\mathrm{T}}} \omega_{n} \mathbf{z}_{n} \mathbf{z}_{n}^{H}, \quad \text { where } \sum_{n=1}^{N_{\mathrm{T}}} \omega_{n}=1, \omega_{n} \geq 0, \omega_{n} \in \mathbb{R}, \forall n,
$$

and $\mathbb{R}$ denotes the set of real numbers. As a result, we can rewrite $\mathbf{0} \in \partial_{\mathbf{W}_{1}} \mathcal{L}(\boldsymbol{\Xi})$ as $\mathbf{0}=\partial_{\mathbf{W}_{1}} \mathcal{L}(\boldsymbol{\Xi})$ by applying (25) to $\partial_{\mathbf{W}_{1}} \mathcal{L}(\boldsymbol{\Xi})$ which can be expressed as

$$
\begin{aligned}
\mathbf{Y}_{1}^{*} & =\mathbf{I}_{N_{\mathrm{T}}}+\phi^{*} \sum_{n=1}^{N_{\mathrm{T}}} \omega_{n} \mathbf{z}_{n} \mathbf{z}_{n}^{H}-\sum_{k \in \mathcal{P}} \frac{\mu_{1, k}^{*} \mathbf{H}_{k}}{\Gamma_{\mathrm{req}_{1}}} \\
& -\sum_{k \in \mathcal{B}} \frac{\beta_{k}^{*} \mathbf{H}_{k}}{\Gamma_{\mathrm{req}_{1}}}-\sum_{k \in \mathcal{I}} \delta_{k}^{*} \mathbf{H}_{k} .
\end{aligned}
$$

Then, we post-multiply both sides of (26) by $\mathbf{W}_{1}^{*}$ and after exploiting 22 we obtain

$$
\begin{aligned}
& \left(\mathbf{I}_{N_{\mathrm{T}}}+\phi^{*} \sum_{n=1}^{N_{\mathrm{T}}} \omega_{n} \mathbf{z}_{n} \mathbf{z}_{n}^{H}\right) \mathbf{W}_{1}^{*} \\
= & \left(\sum_{k \in \mathcal{P}} \frac{\mu_{1, k}^{*} \mathbf{H}_{k}}{\Gamma_{\mathrm{req}_{1}}}+\sum_{k \in \mathcal{B}} \frac{\beta_{k}^{*} \mathbf{H}_{k}}{\Gamma_{\mathrm{req}_{1}}}+\sum_{k \in \mathcal{I}} \delta_{k}^{*} \mathbf{H}_{k}\right) \mathbf{W}_{1}^{*} .
\end{aligned}
$$

Since $\mathbf{I}_{N_{\mathrm{T}}}+\phi^{*} \sum_{n=1}^{N_{\mathrm{T}}} \omega_{n} \mathbf{z}_{n} \mathbf{z}_{n}^{H}$ is a positive definite matrix, the following equality holds:

$$
\begin{aligned}
& \operatorname{Rank}\left(\mathbf{W}_{1}^{*}\right)=\operatorname{Rank}\left(\left(\mathbf{I}_{N_{\mathrm{T}}}+\phi^{*} \sum_{n=1}^{N_{\mathrm{T}}} \omega_{n} \mathbf{z}_{n} \mathbf{z}_{n}^{H}\right) \mathbf{W}_{1}^{*}\right) \\
= & \operatorname{Rank}\left(\left(\sum_{k \in \mathcal{P}} \frac{\mu_{1, k}^{*} \mathbf{H}_{k}}{\Gamma_{\mathrm{req}_{1}}}+\sum_{k \in \mathcal{B}} \frac{\beta_{k}^{*} \mathbf{H}_{k}}{\Gamma_{\mathrm{req}_{1}}}+\sum_{k \in \mathcal{I}} \delta_{k}^{*} \mathbf{H}_{k}\right) \mathbf{W}_{1}^{*}\right) \\
\leq & \operatorname{Rank}\left(\sum_{k \in \mathcal{P}} \frac{\mu_{1, k}^{*} \mathbf{H}_{k}}{\Gamma_{\mathrm{req}_{1}}}+\sum_{k \in \mathcal{B}} \frac{\beta_{k}^{*} \mathbf{H}_{k}}{\Gamma_{\mathrm{req}_{1}}}+\sum_{k \in \mathcal{I}} \delta_{k}^{*} \mathbf{H}_{k}\right) \leq K .
\end{aligned}
$$

Thus, $\operatorname{Rank}\left(\mathbf{W}_{1}^{*}\right) \leq \min \left\{K, N_{\mathrm{T}}\right\}$. On the other hand, a rank reduction approach can be used to show $\sum_{l=1}^{L} \operatorname{Rank}^{2}\left(\mathbf{W}_{l}^{*}\right)+$ $\operatorname{Rank}^{2}\left(\mathbf{W}_{\mathrm{E}}^{*}\right) \leq \operatorname{card}(\mathcal{P}) L+\operatorname{card}(\mathcal{B})+\operatorname{card}(\mathcal{I})$ for $\phi=0$. Please refer to [24, Theorem 3.2] for a detailed proof.

\section{REFERENCES}

[1] Q. Li and W.-K. Ma, "Multicast Secrecy Rate Maximization for MISO Channels with Multiple Multi-Antenna Eavesdroppers," in Proc. IEEE Intern. Commun. Conf., Jun. 2011, pp. 1-5.

[2] N. Sidiropoulos, T. Davidson, and Z.-Q. Luo, "Transmit Beamforming for Physical-Layer Multicasting," IEEE Trans. Signal Process., vol. 54, pp. 2239-2251, Jun. 2006.

[3] D. W. K. Ng, E. Lo, and R. Schober, "Energy-Efficient Resource Allocation in OFDMA Systems with Large Numbers of Base Station Antennas," IEEE Trans. Wireless Commun., vol. 11, pp. 3292-3304, Sep. 2012.

[4] L. Varshney, "Transporting Information and Energy Simultaneously," in Proc. IEEE Intern. Sympos. on Inf. Theory, Jul. 2008, pp. $1612-1616$.

[5] P. Grover and A. Sahai, "Shannon Meets Tesla: Wireless Information and Power Transfer," in Proc. IEEE Intern. Sympos. on Inf. Theory, Jun. 2010, pp. $2363-2367$

[6] R. Zhang and C. K. Ho, "MIMO Broadcasting for Simultaneous Wireless Information and Power Transfer," IEEE Trans. Wireless Commun., vol. 12, pp. 1989-2001, May 2013.

[7] D. W. K. Ng, E. S. Lo, and R. Schober, "Wireless Information and Power Transfer: Energy Efficiency Optimization in OFDMA Systems," IEEE Trans. Wireless Commun., vol. 12, pp. 6352 - 6370, Dec. 2013.

[8] C. Christopoulos, A. Skodras, and T. Ebrahimi, "The JPEG2000 Still Image Coding System: An Overview," IEEE Trans. Consum. Electron., vol. 46, pp. 1103-1127, Nov. 2000.

[9] H. Schwarz, D. Marpe, and T. Wiegand, "Overview of the Scalable Video Coding Extension of the H.264/AVC Standard,” IEEE Trans. Circuits Syst. Video Technol., vol. 17, pp. 1103-1120, Sep. 2007.

[10] A. D. Wyner, "The Wire-Tap Channel," Tech. Rep., Oct. 1975.

[11] S. Goel and R. Negi, "Guaranteeing Secrecy using Artificial Noise," IEEE Trans. Wireless Commun., vol. 7, pp. 2180-2189, Jun. 2008.

[12] D. W. K. Ng, E. S. Lo, and R. Schober, "Secure Resource Allocation and Scheduling for OFDMA Decode-and-Forward Relay Networks," IEEE Trans. Wireless Commun., vol. 10, pp. 3528-3540, Aug. 2011.

[13] —, "Robust Beamforming for Secure Communication in Systems with Wireless Information and Power Transfer," submitted for possible publication, Nov. 2013. [Online]. Available: http://arxiv.org/abs/1311.2507

[14] Y. Fallah, H. Mansour, S. Khan, P. Nasiopoulos, and H. Alnuweiri, "A Link Adaptation Scheme for Efficient Transmission of H.264 Scalable Video Over Multirate WLANs," IEEE Trans. Circuits Syst. Video Technol., vol. 18, pp. 875-887, Jun. 2008.

[15] B. Barmada, M. Ghandi, E. Jones, and M. Ghanbari, "Prioritized Transmission of Data Partitioned H.264 Video with Hierarchical QAM," IEEE Signal Process. Lett., vol. 12, pp. 577-580, Aug. 2005.

[16] Q. Wang, H. Su, K. Ren, and K. Kim, "Fast and Scalable Secret Key Generation Exploiting Channel Phase Randomness in Wireless Networks," in Proc. IEEE Intern. Conf. on Comput. Commun., 2011, pp. 1422-1430.

[17] D. Tse and P. Viswanath, Fundamentals of Wireless Communication, 1st ed. Cambridge University Press, 2005.

[18] S. Boyd and L. Vandenberghe, Convex Optimization. Cambridge University Press, 2004.

[19] J. F. Sturm, "Using SeDuMi 1.02, A MATLAB Toolbox for Optimization over Symmetric Cones," Optimiz. Methods and Software, vol. 11-12, pp. 625-653, Sep. 1999.

[20] IEEE P802.11 Wireless LANs, “TGn Channel Models", IEEE 802.1103/940r4, Tech. Rep., May 2004.

[21] H.-S. Chen and W. Gao, "MAC and PHY Proposal for 802.11af," Tech. Rep., Feb., [Online] https://mentor.ieee.org/802.11/dcn/10/11-10-0258-00-00af-mac-and-phy-proposal-for-8

[22] J. B. Lasserre, "A Trace Inequality for Matrix Product," IEEE Trans. Automatic Control, vol. 40, pp. 1500-1501, Aug. 1995.

[23] A. S. Lewis, "Convex Analysis on the Hermitian Matrices," Soc. for Ind. and Appl. Mathematics (SIAM) J. on Optimization, vol. 6, pp. 164-177, 1996.

[24] Y. Huang and D. Palomar, "Rank-Constrained Separable Semidefinite Programming With Applications to Optimal Beamforming," IEEE Trans. Signal Process., vol. 58, pp. 664-678, Feb. 2010. 